\title{
Pinosylvin exacerbates LPS-induced apoptosis via ALOX 15 upregulation in leukocytes
}

\author{
Ohseong Kwon, Youngsik Seo E Heonyong Park ${ }^{*}$ \\ Department of Molecular Biology \& Institute of Nanosensor and Biotechnology, Dankook University, Cheonan 31116, Korea
}

Pinosylvin is known to have anti-inflammatory activity in endothelial cells. In this study, we found that pinosylvin had a pro-apoptotic activity in lipopolysaccharide (LPS)-preconditioned leukocytes. This finding suggests that pinosylvin has an effect on the resolution of inflammation. To understand the detailed mechanism, we examined if pinosylvin enhances cyclooxygenase (COX) or lipoxygenase (LOX) activity in THP-1 and U937 cells. LOX activity was found to be markedly increased by pinosylvin, whereas COX activity was not altered. Furthermore, we found that pinosylvin enhanced both levels of ALOX 15 mRNA and protein, implying that LOX activity, elevated by pinosylvin, is attributed to upregulation of ALOX 15 expression. From this cell signaling study, pinosylvin appeared to promote phosphorylations of ERK and JNK. ERK or JNK inhibitors were found to attenuate ALOX 15 expression and LPS-induced apoptosis promoted by pinosylvin. In conclusion, pinosylvin enhances the apoptosis of LPSpreconditioned leukocytes by up-regulating ALOX 15 expression through ERK and JNK. These findings suggest that pinosylvin may induce the resolution of inflammation. [BMB Reports 2018; 51(6): 302-307]

\section{INTRODUCTION}

Inflammatory processes are essential to primary lines of defense. However, dysregulated, excessive, or prolonged inflammation will promote a variety of pathological conditions which include ischemia-reperfusion, allergy, carcinoma, lupus, and the respiratory distress syndrome (1-5). Uncontrolled activation of leukocytes induces pathological conditions through cytotoxic substances such as various pro-inflammatory agents and reactive oxygen species (ROS). Therefore, inflammation has to be appropriately terminated.

*Corresponding author. Tel: +82-41-550-3489; Fax: +82-41-5597941; E-mail: heonyong@dankook.ac.kr

https://doi.org/10.5483/BMBRep.2018.51.6.024

Received 3 February 2018, Revised 22 February 2018, Accepted 19 March 2018

Keywords: ALOX 15, Apoptosis, Leukocyte, Lipoxygenase, Pinosylvin
Resolution of inflammation is found to be mediated by compounds produced by leukocytes at the onset and at the progression stages of inflammation (6). Compounds that can induce the resolution of inflammation include lipoxins, resolvins, and protectins. Lipoxins, resolvins and protectins are produced by lipoxygenase (LOX). The resolution of inflammation is not due to the spontaneous removal of inflammatory process. Programmed processes, including LOX activation, are involved in the resolution of inflammation instead. Activation of the resolution process can prevent excessive and persistent inflammation, thereby reducing prolonged exposure of pro-inflammatory agents to adjacent tissues or cells. Dysregulated resolution of inflammation is also responsible for chronic inflammatory diseases. In this respect, the resolution of the inflammatory process plays a key role in the prevention of inflammatory diseases. Although mechanisms involved in the resolution of inflammation have been intensively studied, they have not been fully elucidated.

Reducing the activity of leukocytes or enhancing cell death of leukocytes has been suggested to modulate chronic inflammatory diseases. Phytochemicals which can control the resolution of inflammation can be utilized as pharmacological drugs for chronic inflammatory diseases (7). Pinosylvin (3,5-dihydroxy-trans-stilbene) is a naturally occurring stilbenoid found in leaves or woods of various Pinus species. Several lines of evidence have shown that pinosylvin exerts multiple cellular functions which include cell proliferation, antioxidant and anti-tumoric activity (8-10). Although extensive works have been undertaken, cell type-specific effects of pinosylvin remain controversial. Moreover, its mechanisms of action have not been fully investigated. In the vascular system, pinosylvin, at concentrations higher than $100 \mu \mathrm{mol} / \mathrm{L}$, can induce cell death, including apoptosis and autophagy. Vascular cell death is proposed to cause cardiovascular diseases, including myocardial infarction (11). However, at lower concentrations $(\leq 1 \mu \mathrm{mol} / \mathrm{L})$, pinosylvin can promote angiogenesis, cell proliferation and anti-adhesiveness (8). In addition, short-term (10 min) exposure of leukocytes to pinosylvin can inhibit the oxidation burst and neutrophil activation, while it has no effect on apoptosis $(7,12,13)$. Taken together, we hypothesized that pinosylvin at lower concentrations plays a part in the immune responses in leukocytes.

ISSN: 1976-670X (electronic edition)

Copyright (c) 2018 by the The Korean Society for Biochemistry and Molecular Biology

(c) This is an open-access article distributed under the terms of the Creative Commons Attribution Non-Commercial License (http://creativecommons.org/licenses/by-nc/4.0) which permits unrestricted non-commercial use, distribution, and reproduction in any medium, provided the original work is properly cited. 
To study the effects of pinosylvin on leukocytes, we utilized THP-1 and U937 cells, as they are the most frequently used cells for this purpose $(14,15)$. THP-1 and U937 cells are human monocytic leukemia and myeloid leukemia cells, respectively, that have monocytic entities. Moreover these cells are commonly used to estimate the anti-leukemia efficacy of phytochemicals in a variety of laboratories. Accordingly, these cell lines are established tools to test the effects of pinosylvin on the pathophysiology of leukocytes.

The objective of this study was to determine whether pinosylvin could induce immune responses, such as resolution of inflammation, and how pinosylvin could modulate those responses in leukocytes. The results of this study will provide further insight into the pharmacological effects of pinosylvin on immunological diseases.

\section{RESULTS}

\section{Pinosylvin exacerbates lipopolysaccharide-triggered apoptosis in the leukocyte}

We first measured cytotoxic activity of pinosylvin at various concentrations. As displayed in Fig. 1A, pinosylvin had no cytotoxic effect on leukocytes at $\leq 10 \mu \mathrm{mol} / \mathrm{L}$, indicating that $0.1 \mu \mathrm{mol} / \mathrm{L}$ of pinosylvin can be safely used for pharmaceutical purposes. Then we tested the pro- or anti-apoptotic activity of
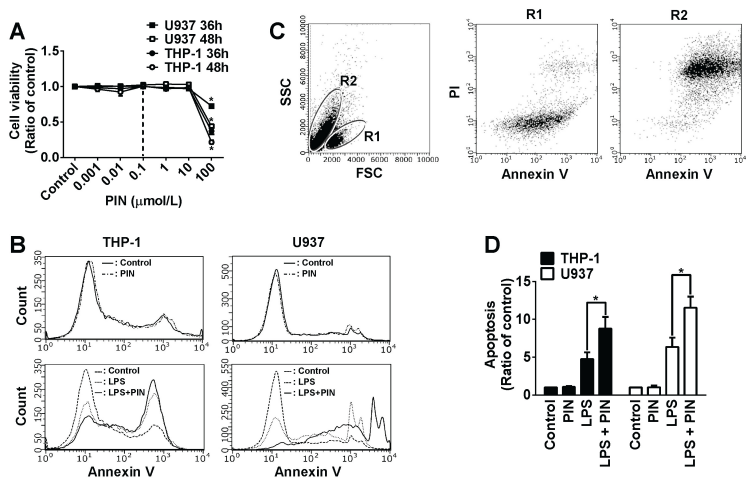

Fig. 1. Pinosylvin exacerbates apoptosis in lipopolysaccharide (LPS)-preconditioned leukocytes. (A) THP-1 and U937 cells were incubated with various concentrations of pinosylvin. Then cytotoxic activity was measured by Trypan blue staining. Line graphs represent the percentage of dead cells (means \pm S.E., $n$ $=3$ ). $* P<0.05$. (B) THP-1 and U937 cells preconditioned with $10 \mu \mathrm{g} / \mathrm{ml}$ lipopolysaccharide (LPS) for $16 \mathrm{~h}$ in RPMI 1640 containing none or $0.1 \mu \mathrm{mol} / \mathrm{L}$ of pinosylvin (PIN) were incubated with Annexin V-FITC and propidium iodide (PI). Cells were then subjected to flow cytometry analysis. (C) Representitive plots of U937 cells assayed by flow cytometry. Cells were sorted and located in the R1 and R2 areas by light scattering. Cells in R1 and R2 are viable (Annexin V/PI-unstained) and apoptotic (or necrotic, Annexin V/PI-stained), respectively. Apoptotic cells were counted and plotted in panel (D). The bar graphs represent the percentages of apoptotic cells (means \pm S.E., $\mathrm{n}=3$ ). $* \mathrm{P}<$ 0.05 .
$0.1 \mu \mathrm{mol} / \mathrm{L}$ pinosylvin by utilizing flow cytometry (Fig. 1B, C). We obtained two forms of THP-1 cells separated in R1 and R2 area by flow cytometry, when cells were treated with LPS (Fig. 1C). Cells in R1 appeared to be viable (Annexin V/PIunstained), whereas cells in R2 were shown to be mostly apoptotic (Annexin V/PI-stained). Based on this finding, the percent of apoptosis was calculated as (the number of cells in R2) / (the number of cells in R1 + R2) $\times 100$. From this assay, it was found that a single treatment of pinosylvin at $0.1 \mu \mathrm{mol} / \mathrm{L}$ had no effect on leukocytic cell apoptosis, compared to the untreated control (Fig. 1B). This result was consistent with non-cytotoxic effects of $0.1 \mu \mathrm{mol} / \mathrm{L}$ pinosylvin. Moreover, pinosylvin at $0.1 \mu \mathrm{mol} / \mathrm{L}$ appeared to be pro-apoptotic in leukocytes pre-conditioned by lipopolysaccharide (LPS). As shown in Fig. 1B, D, pinosylvin exacerbated LPS-induced apoptosis by $\sim 180 \%$ and $\sim 170 \%$ in THP-1 and U937 cells, respectively. These findings imply that pinosylvin may promote leukocytic cell death when leukocytes are infected and inflamed by pathogens. Apoptosis of inflamed leukocytes is likely to be a process of the resolution of inflammation (16).

\section{Pinosylvin activates lipoxygenase in leukocytes}

It is well established the resolution of inflammation is triggered by some eicosanoids such as: lipoxins, resolvins, and protectins (17) Pro-resolving eicosanoids are generated by lipoxygenases (LOX) or cyclooxygenases (COX). To confirm whether eicosanoids are involved in pinosylvin-induced apoptosis and to investigate the detailed mechanism, we first tested whether pinosylvin can activate COX and LOX. Activities of COX-1 and COX-2 were not changed in any significant way by treatment with various concentrations
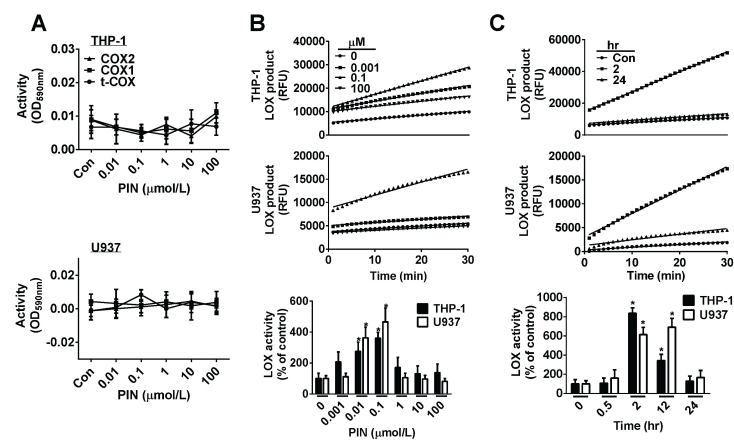

Fig. 2. Pinosylvin activates lipoxygenase (LOX), but does not affect the activity of cyclooxygenase (COX) in THP-1 and U937 cells. (A) The activity of COX-1 or -2 was not altered by 0.1 $\mu \mathrm{mol} / \mathrm{L}$ of pinosylvin (PIN) in both THP-1 and U937 cells. (B) Kinetics of LOX was obtained according to Provider's mannual in THP-1 and U937 cells treated for 2 hrs with various concentrations of pinosylvin (top panel). The bar graph in the bottom panel indicates dose-dependent activities (means \pm S.E., $n$ $=3$ ). ${ }^{*} P<0.05$. (C) The bar graph in the bottom panel shows the change in LOX activity for time-course treatment with 0.1 $\mu \mathrm{mol} / \mathrm{L}$ of PIN (means \pm S.E., $\mathrm{n}=3$ ). ${ }^{*} \mathrm{P}<0.05$ 
(0-100 $\mu \mathrm{mol} / \mathrm{L}$ ) of pinosylvin in THP-1 and U937 cells (Fig. 2A). However, LOX activity was remarkably increased by pinosylvin in both THP-1 and U937 cells (Fig. 2B, C). LOX activity was maximally enhanced by 4 - and 5 -fold in THP-1 and U937 cells, respectively, when cells were treated with 0.1 $\mu \mathrm{mol} / \mathrm{L}$ of pinosylvin. Time-course experiment appeared to be maximal at a $2 \mathrm{hr}$ exposure and then LOX activation was reduced by longer exposures ( $\geq 12 \mathrm{hr}$ ) in THP-1 cells. In U937 cells, maximally increased LOX activity remained for up to $12 \mathrm{hrs}$ and then decreased at $24 \mathrm{hr}$ exposure. Conclusively, pinosylvin temporarily activated LOX in leukocytes, but not COX.

\section{Pinosylvin promotes expression of ALOX 15 in leukocytes}

The human genome possesses six isotype genes of LOX, such as ALOX5, ALOX12, ALOX12B, ALOX15, ALOX15B, and ALOXE3 (17). Pre-resolving eicosanoids are known to be produced by ALOX5, ALOX12 and ALOX15 (17, 18). Therefore, we tested if pinosylvin has any effect on the gene expression of ALOX5, ALOX12 and ALOX15. Transcripts for three of these genes were measured by real-time PCR. As shown in Fig. 3A, the transcription of ALOX15 was remarkably elevated by 5 times, when THP-1 and U937 cells were treated for $30 \mathrm{~min}$ with $0.1 \mu \mathrm{mol} / \mathrm{L}$ of pinosylvin. The amount of
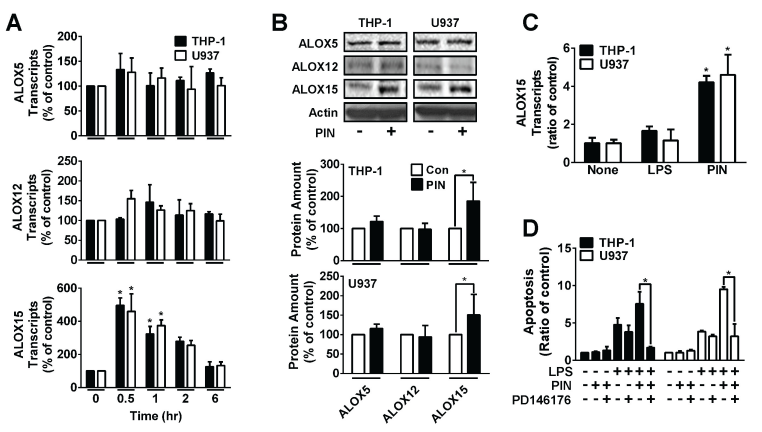

Fig. 3. Pinosylvin promotes ALOX15 expression. (A) THP-1 and U937 cells were incubated for various periods with $0.1 \mu \mathrm{mol} / \mathrm{L}$ of pinosylvin (PIN). Then transcripts of three different types of ALOX were measured by real-time PCR. Bar graphs show the time-course of mRNA change (means \pm S.E., $\mathrm{n}=3$ ). $* \mathrm{P}<$ 0.05. (B) THP-1 and U937 cells were treated for $1 \mathrm{hr}$ with or without $0.1 \mu \mathrm{mol} / \mathrm{L}$ of PIN. After cells were lysed, proteins in cell lysates were resolved by SDS-PAGE, transferred to PVDF membranes, and immunoblotted with indicated antibody. Data were quantified using a densitometry. Quantified data were shown in the bottom panel (means \pm S.E., $n=3$ ). ${ }^{*} P<0.05$. (C) THP-1 and U937 cells were treated with or without $10 \mu \mathrm{g} / \mathrm{ml}$ of lipopolysaccharide (LPS) and $0.1 \mu \mathrm{mol} / \mathrm{L}$ of PIN. Then transcripts of ALOX15 were measured as previously described in panel A. Data were shown as the bar graph (means \pm S.E., $n=$ 3). $* P<0.05$. (D) THP-1 and U937 cells were pre-treated with or without $10 \mu \mathrm{g} / \mathrm{ml}$ of LPS and/or $10 \mu \mathrm{mol} / \mathrm{L}$ of PD146176, 2 hrs before treating with PIN. Then cell apoptosis was analyzed as described in Fig. 1. The bar graph indicates cell apoptosis (means \pm S.E., $\mathrm{n}=3$ ). $* \mathrm{P}<0.05$. transcripts of ALOX5 and ALOX12 were not significantly changed by treatment with pinosylvin. In addition, transcript of ALOX15-2 was not detected in THP-1 and U937 cells. This result was confirmed by Western blots. Only for ALOX15, the protein level was elevated by treatment with $0.1 \mu \mathrm{mol} / \mathrm{L}$ of pinosylvin, but not for ALOX5 and ALOX12 (Fig. 3B). Intriguingly, LPS had no effect on the transcription of ALOX15 (Fig. 3C), indicating that LPS-induced apoptosis is not associated with ALOX15. In conclusion, pinosylvin enhances the amount of mRNA and protein of ALOX15. Then, to learn whether ALOX15 is directly involved in pinosylvin-exacerbated leukocytic apoptosis, a pharmaceutical assay utilizing an ALOX15 inhibitor was executed. When THP-1 and U937 cells were pretreated with PD146176, known as an ALOX15 inhibitor before treating with pinosylvin, the synergistic pro-apoptotic activity of pinosylvin completely disappeared (Fig. 3D). However, PD146176 had no effect on LPS-induced apoptosis. Therefore, this result indicates that ALOX15 is directly involved in the pinosylvin-exacerbated leukocytic apoptosis.

\section{Pinosylvin-promoted ALOX15 expression and LPS-preconditioned apoptosis were mediated by activation of ERK and JNK}

We went on to test if pinosylvin activated mitogen activated protein kinases (MAPK) and/or IKB. As displayed in Fig. 4A, B, pinosylvin at $0.1 \mu \mathrm{mol} / \mathrm{L}$ elevated phosphorylations of
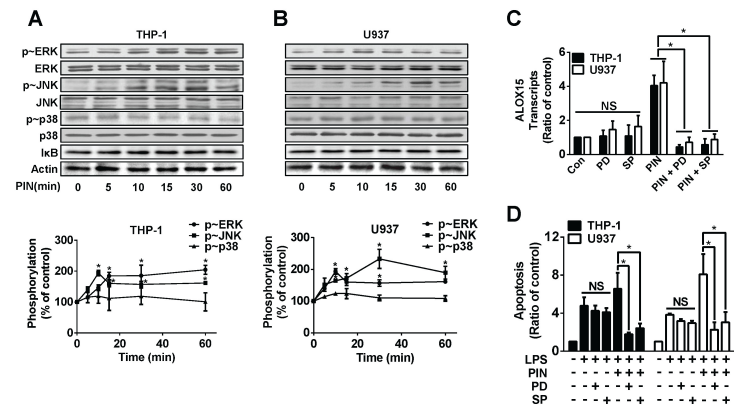

Fig. 4. ERK and JNK mediates pinosylvin-prompted ALOX15 expression and apoptosis in leukocytes. THP-1 (A) and U937 (B) cells were treated with or without $0.1 \mu \mathrm{mol} / \mathrm{L}$ pinosylvin for indicated time period, harvested, and lysed. Western blot analysis for proteins in cell lysates using different antibodies. Representative plots are shown from experiments performed in triplicates. In bottom panel, Western blot results were scanned by densitometry and quantified. Bar graphs represent means \pm S.E. $(\mathrm{n}=3)$. ${ }^{*} \mathrm{P}<0.05$. (C) THP-1 and U937 cells were pretreated with or without PD98059 (50 $\mu \mathrm{mol} / \mathrm{L})$ and/or SP600125 (50 $\mu \mathrm{mol} / \mathrm{L}) 1 \mathrm{hr}$ before treating PIN. Then transcripts of ALOX15 were measured as previously described in Fig. 3. Data were shown as the bar graph (means \pm S.E., $n=3$ ). $* P<0.05, N S$ : not significant. (D) THP-1 and U937 cells were pretreated with or

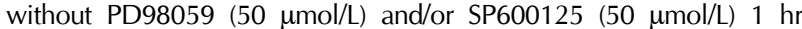
before treating PIN. The bar graph indicates cell apoptosis (means \pm S.E., $\mathrm{n}=3$ ). ${ }^{*} \mathrm{P}<0.05$, NS: not significant. 
extracellular signal regulated kinase (ERK) and cJun $\mathrm{N}$-terminal kinase (JNK), whereas pinosylvin had no effects on p38 MAPK and IKB. In both THP-1 and U937 cells, ERK and JNK phosphorylation was maximally increased by $\sim 2$-fold when cells were incubated for $10 \mathrm{~min}$ with $0.1 \mu \mathrm{mol} / \mathrm{L}$ of pinosylvin. Then, we determined whether ERK and JNK activations, induced by pinosylvin, played an important role in ALOX15 expression and LPS-triggered apoptosis. When cells were pretreated with PD98059 (an ERK inhibitor) or SP600125 (a JNK inhibitor), pinosylvin-induced promotion of ALOX15 transcript disappeared (Fig. 4C). In addition, ERK and JNK inhibitors partly reversed pinosylvin-exacerbated apoptosis in LPS-preconditioned cells (Fig. 4D). Therefore, we concluded that pinosylvin-controlled expression of ALOX15 and apoptosis are mediated by ERK and JNK.

\section{DISCUSSION}

A recent strategy for drug discovery, to treat inflammatory diseases, is by screening drugs that increase the resolution of inflammation or have better anti-inflammatory functions. Resolution of inflammation suppresses the expression of pro-inflammatory genes and promotes pro-inflammatory cell death, thereby repairing inflamed tissues. This repair returns and maintains a small population of monocytes $(19,20)$. Prostaglandins, stimulants for signaling inflammation resolution, are created at the initial stage of inflammation (21). Prostaglandins $E_{2}$ and $D_{2}$ then stimulate transcriptional activity of lipoxins, resolvins, and protectins, the dominant activators for inflammation resolution (20, 22-24). Effective elimination of leukocytes at the cellular level is a necessity to achieve inflammation resolution. It is currently accepted that the elimination of leukocytes is initiated by leukocytic apoptosis and then cleared by macrophage-mediated phagocytosis. Bridging molecules, such as thrombospondin 1, integrin $\alpha_{w} \beta_{3}$, CD36, and phosphatidylserine receptor(s) are involved in phagocytosis via binding between apoptotic cells and the phagocytes (19). Given that cell death of leukocytes is a prerequisite for the resolution of inflammation (16), our findings concerning pinosylvin-induced leukocytic cell death imply that pinosylvin could be used for the resolution of inflammation.

Human genome contains six isotypes of LOX such as ALOX5, ALOX12, ALOX12B, ALOX15, ALOX15B, and ALOXE3 (17). Additionally, several lines of evidence suggest that ALOX15 produces a strong resolving factor $(16,17)$. ALOX 15 and LOX12/15, murine ortholog of of human ALOX15, are evolutionarily highly conserved and encoded by the ALOX15 gene (25). However ALOX15 expression was reported several years ago, the functional role of human ALOX15 has not been elucidated. Compared with human ALOX15, recent lines of evidence show that murine LOX12/15 exerts pro-resolving functions. Firstly, resolvins, protectins and lipoxins generated by LOX12/15 have anti-inflammatory and pro-resolving activity (25). Secondly, LOX 12/15-deficient mice exhibit enhanced pro-resolving activity, e.g., orchestrated clearance of apoptotic cells (16). Therefore, the present study supports the notion that ALOX15 up-regulation, induced by pinosylvin, may play an important role in the resolution of inflammation. Even so, pinosylvin will be carefully utilized as a resolving drug, because 12-hydroxyicosatetraenoic acid (12-HETE) generated by LOX12/15 has either pro-inflammatory or anti-inflammatory functions (25).

In this study, it was found that the ALOX15 expression was mediated by activation of both ERK and JNK. To date, it is unclear how ERK and JNK promote the expression of ALOX15. It was previously reported that expression of LOX12/15 is promoted by IL-4 and IL-13 $(16,17)$. Based on these findings, it is highly possible that ERK and JNK promote the expression of LOX12/15 by their functional association with IL-4 and IL-13 (26). Detailed mechanisms for this transcriptional regulation remain to be further elucidated.

Atherosclerosis, an inflammatory disease, is initiated by adhesion between leukocytes and endothelial cells. Adhesive leukocytes are then infiltrated into tunica intima. They will proliferate and alter into foam cells which will produce cytokines to recruit smooth muscle cells (27). The proliferation of smooth muscle cells increases the thickening of intima. During these processes, inflammation is a major factor that contributes to atherosclerosis. Based on these processes, the finding that pinosylvin can induce leukocytic cell death suggests that pinosylvin could have anti-atherogenic activity. In this respect, our findings suggest that pinosylvin might have potential as a pharmaceutical drug for cardiovascular diseases including atherosclerosis. Moreover, previous reports utilizing oral intake of resveratrol, a well-known stilbene, appears to increase the concentration up to $50 \mathrm{nmol} / \mathrm{L}-2 \mu \mathrm{mol} / \mathrm{L}$ at plasma level (28-30). Therefore, since the effective dose $(0.1 \mu \mathrm{mol} / \mathrm{L})$ of pinosylvin can be feasible for pharmaceutical application, pinosylvin will be useful as a resolving agent.

\section{MATERIALS AND METHODS}

\section{Cell culture, reagents, and treatment}

Human monocytic cell lines THP-1 and U937 cells originated from acute monocytic leukemia and myelomonocytic lymphoma, respectively. THP-1 and U937 cells were cultured in Roswell Park Memorial Institute Medium 1640 (RPMI 1640, Welgene, Seoul, Korea) supplemented with $10 \%$ fetal bovine serum (FBS, Welgene), $2 \mathrm{mmol} / \mathrm{L}$ L-glutamine, $50 \mu \mathrm{g} / \mathrm{ml}$ penicillin, and $50 \mu \mathrm{g} / \mathrm{ml}$ streptomycin. THP-1 and U937 cells were grown in a humidified incubator with $5 \% \quad \mathrm{CO}_{2}$ atmosphere at $37^{\circ} \mathrm{C}$. These cells were then starved for $12 \mathrm{~h}$ in starvation media (RPMl 1640 supplemented with $50 \mu \mathrm{g} / \mathrm{ml}$ penicillin and $50 \mu \mathrm{g} / \mathrm{ml}$ streptomycin). Various concentrations $(0-100 \mu \mathrm{mol} / \mathrm{L})$ of pinosylvin were used to obtain a dose-curve, while $0.1 \mu \mathrm{mol} / \mathrm{L}$ of pinosylvin was used for a time-course experiment. We also treated cells with different inhibitors 
when necessary. Those inhibitors included PD146176 (an ALOX15 inhibitor; $10 \mu \mathrm{mol} / \mathrm{L}$; Enzo life sciences, New York city, NY, USA), PD98059 (an ERK inhibitor; $50 \mu \mathrm{mol} / \mathrm{L}$, Enzo life sciences) and SP600125 (a JNK inhibitor; $50 \mu \mathrm{mol} / \mathrm{L}$, Enzo life sciences). They were utilized for pretreatment for $1 \mathrm{~h}$ before treatment with pinosylvin.

\section{Fluorescence-activated cell sorting analysis}

Cells were pretreated as aforementioned. Cells were then washed twice with phosphate buffered saline (PBS) and harvested by centrifugation $(1,000 \times \mathrm{g}, 5 \mathrm{~min})$. These harvested cells were suspended in a binding buffer (BioBud, Seongnam, Korea). Cells were stained with both Annexin V-FITC and propidium iodide (PI) using ApoScan Annexin V FITC Apoptosis Detection Kit (BioBud) to detect apoptotic and necrotic cells. Distribution of cells, through apoptosis and post-apoptosis (necrosis), were analyzed by flow cytometry using Guava easyCyte system (Millipore, Billerica, MA, USA). To analyze post-apoptosis (necrosis), specific area was drawn on a cytogram. The area with annexin $\mathrm{V}^{+}$(annexin V-stained cells) and $\mathrm{Pl}^{+}$(Pl-stained cells) was set as post-apoptotic (necrotic) population.

\section{Western blotting}

Proteins (25 $\mu \mathrm{g}$ of soluble lysates) were resolved by SDS-PAGE, transferred to polyvinylidenedifluoride (PVDF) membrane (Millipore), and blotted with antibodies specific to p-ERK (Cell Signaling, Danvers, MA, USA), ERK (Cell Signaling), p-p38 MAP kinase (Cell Signaling), p38 MAP kinase (Cell Signaling), p-JNK (Cell Signaling), JNK (Cell Signaling), I B $\alpha$ (Cell Signaling), ALOX5 (Abcam, Cambridge, MA, USA), ALOX12 (Abcam), ALOX15-1 (Abcam), ALOX15-2 (Abcam) and actin (Santa Cruz Biotechnology, Santa Cruz, CA, USA) (31).

\section{Measurement of lipoxygenase and cyclooxygenase}

The enzyme activities for lipoxygenase (LOX) and cyclooxygenase (COX) were measured using LOX (Biovision, San Francisco bay, CA, USA) and COX (Cayman Chemical, Ann Arbor, MI, USA) assay kits. For dose-curve experiments, THP-1 and $U 937$ cells $\left(4 \times 10^{5}-1 \times 10^{6}\right.$ cells $\left./ \mathrm{ml}\right)$ were treated with a variety of concentrations $(0-100 \mu \mathrm{mol} / \mathrm{L})$ of pinosylvin for 12 hrs, and then the cell lysates were obtained. Cell lysates were prepared by sonication and centrifugation $(16,000 \times \mathrm{g}, 15$ min). LOX and COX activities for cell lysates equivalent to 10 $\mu \mathrm{g}$ of protein were measured according to protocols supplied by manufacturers. Briefly, equal amount of substrate was added to the sample containing cell lysate and then reacted for $5 \mathrm{~min}$ at $25^{\circ} \mathrm{C}$ to analyze COX activity. Then, absorbance at $590 \mathrm{~nm}$ was measured utilizing a spectrophotometer (BioTek, Winooski, VT, USA). In the case of LOX analysis, fluorescence (Ex: $500 \mathrm{~nm}, \mathrm{Em}: 536 \mathrm{~nm}$ ) was measured by the spectrophotometer immediately after adding LOX substrates into the lysate aliquot.

\section{Real-time qPCR analysis}

THP-1 and U937 cells were treated with $0.1 \mu \mathrm{mol} / \mathrm{L}$ of pinosylvin. Then, the total RNA was extracted using QIAzol Lysis Reagent (Qiazen, Hilden, NRW, Germany) and then used for reverse transcription with M-MLV Reverse Transcriptase (Promega, Madison, WI, USA). The reverse transcript was then enhanced with SYBR polymerase mix (Intron, Seongnam, Korea) by using the LightCycler (Roche, Basel, Switzerland) under the following conditions; denatureation at $98^{\circ} \mathrm{C}$ for $30 \mathrm{~s}$, annealing at $61^{\circ} \mathrm{C}$ for $30 \mathrm{~s}$, and extension at $72^{\circ} \mathrm{C}$ for $30 \mathrm{~s}(50$ cycle) (32).

Primers were used as follows;

ALOX5 gene:

sense, 5'-TCTACTACGAGGGCGACCAGG-3'; antisense, 5'-AGATGGCCACACTGTTCGGAA-3'. ALOX12 gene: sense, 5'-CTCTGCCATTTCCTCACCAT-3'; antisense, 5'-AAGGAAGATGTGACGATGGC-3'. ALOX15 gene: sense, 5'-ACTGAAATCGGGCTGCAAGGG-3'; antisense, 5'-GGGTGATGGGGGCTGAAATAA-3'. GAPDH gene: sense, 5'-CCATCACCATCTTCCAGGAGCGA-3'; antisense, 5'-GGATGACCTTGCCCACAGCCTTG-3'.

\section{ACKNOWLEDGEMENTS}

This work was supported by 2017 research funds from Dankook University, Republic of Korea.

\section{CONFLICTS OF INTEREST}

The authors have no conflicting interests.

\section{REFERENCES}

1. Ashley NT, Weil ZM and Nelson RJ (2012) Inflammation: Mechanisms, Costs, and Natural Variation. Annu Rev Ecol Evol Syst 43, 385-406

2. Cascão R, Rosário HS and Fonseca JE (2009) Neutrophils: warriors and commanders in immune mediated inflammatory diseases. Acta Reumatol Port 34, 313-326

3. Cascão R, Rosário HS, Fonseca JE et al (2010) Neutrophils in rheumatoid arthritis: More than simple final effectors. Autoimmun Rev 9, 531-535

4. Fialkow L, Wang Y and Downey GP (2007) Reactive oxygen and nitrogen species as signaling molecules regulating neutrophil function. Free Rad Biol Med 42, 153-164

5. Wright HL, Moots RJ, Edwards SW et al (2010) Neutrophil function in inflammation and inflammatory diseases. Rheumatology 49, 1618-1631

6. Clarke M, Bennett M and Littlewood T (2007) Cell death in the cardiovascular system. Heart 93, 659-664

7. Jančinová V, Perečko T, Drábiková K et al (2012) Decreased activity and accelerated apoptosis of 
neutrophils in the presence of natural polyphenols. Interdiscip Toxicol 5, 59-64

8. Jeong $\mathrm{E}$, Lee HR, Park H et al (2013) Pinosylvin induces cell survival, migration and anti-adhesiveness of endothelial cells via nitric oxide production. Phytother Res 27, 610-617

9. Koskela A, Reinisalo M, Karjalainen RO et al (2014) Pinosylvin-mediated protection against oxidative stress in human retinal pigment epithelial cells. Mol Vis 20, 760-769

10. Park EJ, Park HJ, Lee SK et al (2012) Antimetastatic activity of pinosylvin, a natural stilbenoid, is associated with the suppression of matrix metalloproteinases. J Nutr Biochem 23, 946-952

11. Park J, Pyee J and Park H (2014) Pinosylvin at a high concentration induces AMPK-mediated autophagy for preventing necrosis in bovine aortic endothelial cells. Can J Physiol Pharmacol 92, 993-999

12. Jančinová V, Perečko T, Drábiková K et al (2012) The natural stilbenoid pinosylvin and activated neutrophils: effects on oxidative burst, protein kinase $\mathrm{C}$, apoptosis and efficiency in adjuvant arthritis. Acta Pharmacol Sin 33, $1285-1292$

13. Perečko T, Drábiková K, Jančinová V et al (2013) Involvement of caspase-3 in stilbene derivatives induced apoptosis of human neutrophils in vitro. Interdiscip Toxicol 5, 76-80

14. Chanput W, Mes JJ and Wichers HJ (2014) THP-1 cell line: an in vitro cell model for immune modulation approach. Int Immunopharmacol 23, 37-45

15. Strefford JC, Foot NJ, Jones LK et al (2001) The characterisation of the lymphoma cell line U937, using comparative genomic hybridisation and multi-plex FISH. Cytogenet Cell Genet 94, 9-14

16. Uderhardt S, Herrmann M, Krönke G et al (2012) 12/15-lipoxygenase orchestrates the clearance of apoptotic cells and maintains immunologic tolerance. Immunity 36, 834-846

17. Ivanov I, Kuhn H and Heydeck D (2015) Structural and functional biology of arachidonic acid 15-lipoxygenase-1 (ALOX15). Gene 573, 1-32

18. Tam VC (2013) Lipidomic profiling of bioactive lipids by mass spectrometry during microbial infections. Semin Immunol 25, 240-248

19. Serhan CN and Savill J (2005) Resolution of inflammation: the beginning programs the end. Nat Immunol 6, 1191-1197

20. Serhan CN, Hong S, Moussignac RL et al (2002) Resolvins: a family of bioactive products of omega- 3 fatty acid transformation circuits initiated by aspirin treatment that counter pro-inflammation signals. J Exp Med 196, 1025-1037

21. Levy BD, Clish CB, Serhan CN et al (2001) Lipid mediator class switching during acute inflammation: signals in resolution. Nat Immunol 2, 612-619

22. Hong S, Gronert K, Serhan CN et al (2003) Novel docosatrienes and 17S-resolvins generated from docosahexaenoic acid in murine brain, human blood and glial cells: autacoids in anti-inflammation. J Biol Chem 278, 14677-14687

23. Marcheselli VL, Hong S, Bazan NG et al (2003) Novel docosanoids inhibit brain ischemia-reperfusion-mediated leukocyte infiltration and pro-inflammatory gene expression. J Biol Chem 278, 43807-43817

24. Serhan CN, Clish CB, Gronert K et al (2000) Novel functional sets of lipid-derived mediators with antiinflammatory actions generated from omega-3 fatty acids via cyclooxygenase2-nonsteroidal anti-inflammatory drugs and transcellular processing. J Exp Med 192, 1197-1204

25. Ackermann JA, Hofheinz K, Krönke G et al (2017) The double-edged role of 12/15-lipoxygenase during inflammation and immunity. Biochim Biophys Acta 1862, 371-381

26. Roca H, Craig MJ, Pienta KJ et al (2012) IL-4 induces proliferation in prostate cancer PC3 cells under nutrient-depletion stress through the activation of the JNK-pathway and survivin up-regulation. J Cell Biochem 113, 1569-1580

27. Libby P, Ridker PM and Hansson GK (2011) Progress and challenges in translating the biology of atherosclerosis. Nature 473, 317-325

28. Goldberg DM, Yan J and Soleas GJ (2003) Absorption of three wine-related polyphenols in three different matrices by healthy subjects. Clin Biochem 36, 79-87

29. Tomé-Carneiro J, Larrosa M, Espín JC et al (2013) Resveratrol and clinical trials: the crossroad from in vitro studies to human evidence. Curr Pharm Des 19, 6064-6093

30. Walle T, Hsieh F, Walle UK et al (2004) High absorption but very low bioavailability of oral resveratrol in humans. Drug Metab Dispos 32, 1377-1382

31. Lee CS, Ghim J, Song P, Suh PG, Ryu SH (2016) Loss of phospholipase D2 impairs VEGF-induced angiogenesis. BMB Rep 49, 191-196

32. Kim SJ, Cha JY, Kang HS et al (2016) Corosolic acid ameliorates acute inflammation through inhibition of IRAK-1 phosphorylation in macrophages. BMB Rep 49, 276-281 